\title{
Ultrasound_Guided Erector Spinae Plane Block Reduces Postoperative Opioid Consumption Following Breast Surgery: A Randomized Controlled Study
}

\author{
Yavuz Gürkan, Can Aksu, Alparslan Kuş, Ufuk H. Yörükoğlu, Cennet T. Kılıç \\ Department of Anesthesiology and Reanimation, School of Medicine, Kocaeli University, \\ Kocaeli, Turkey
}

Purpose

Postoperative analgesia in breast surgery is a difficult and overworked issue due to extensive surgery and complex innervation of the breast. Erector spinae plane block (ESB) is a new defined regional anesthesia technique for thoracic analgesia. Although there are some case reports about ESB in breast surgeries, there is no published randomized controlled study in the literature about ESB use for this purpose. Main purpose of this study was to evaluate the analgesic effect of ultrasound guided ESB in breast surgery.

\section{Methods}

Fifty ASA I-II female patients, aged 25-65, who were scheduled to go under elective breast surgery were included to the study. Patients were randomized into two group as ESB and Control group. Single shot ultrasound (US) guided ESB with $20 \mathrm{ml} 0.25$ $\%$ bupivacaine was done preoperatively to all patients in ESB group. Patients in both groups were provided with iv patient controlled analgesia device containing morphine for the postoperative analgesia. Morphine consumptions and numeric rating scale (NRS) scores for pain were recorded at $1^{\text {st }}, 6^{\text {th }}, 12^{\text {th }}$ and $24^{\text {th }}$ hours postoperatively.

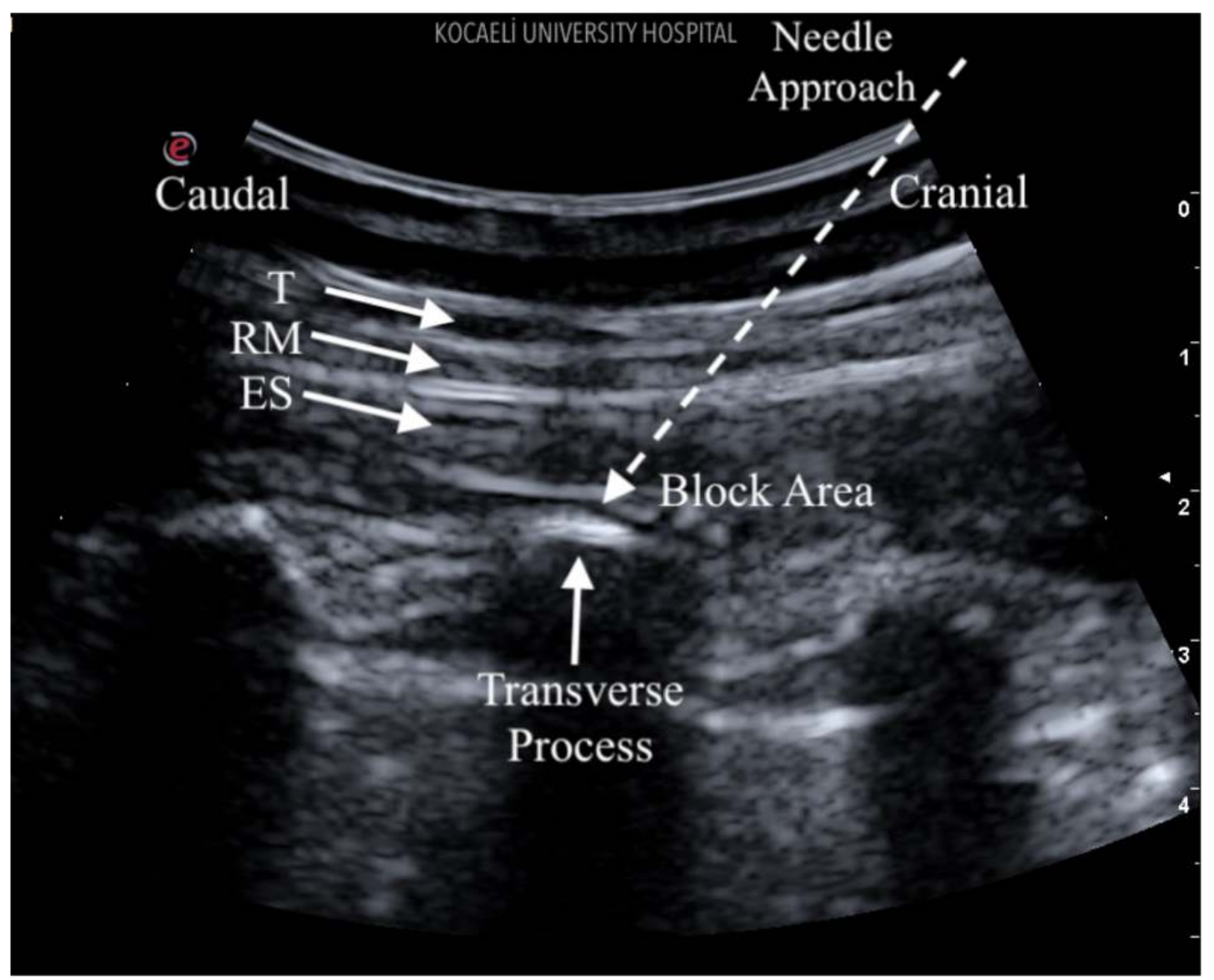

Results

There were no significant differences between ESB and control groups for VAS scores at $1^{\text {st }}, 6^{\text {th }}, 12^{\text {th }}$ and $24^{\text {th }}$ hour (Median VAS values were $2,2,0,0$, and 2, 2, 1, 1 respectively). Postoperative morphine consumptions were significantly lower in ESB group compared to control group at postoperative $1^{\text {st, }} 6^{\text {th }}, 12^{\text {th }}, 24^{\text {th }}$ hour (Median doses of morphine consumptions were 1, 2, 3, 5 and $1,5,10,16 \mathrm{mg}$ respectively) $(p<0,05)$.

\begin{tabular}{|c|c|c|c|}
\hline & $\begin{array}{c}\text { ESB Group } \\
(n=25)\end{array}$ & $\begin{array}{l}\text { Control Group } \\
(\mathbf{n}=\mathbf{2 5})\end{array}$ & $p$ \\
\hline Age (year) & $49.56 \pm 10.96$ & $49.8 \pm 10.49$ & 0.937 \\
\hline Weight (kg) & $72.48 \pm 11.48$ & $73.16 \pm 10.58$ & 0.770 \\
\hline Height $(\mathrm{cm})$ & $161 \pm 5.04$ & $161 \pm 4.55$ & 0.829 \\
\hline BMI (kg/m2) & $27.88 \pm 4.66$ & $28.29 \pm 4.43$ & 0.752 \\
\hline ASA (I/II) & $10 / 15$ & $11 / 14$ & 0.777 \\
\hline Duration of Surgery (min) & $89.4 \pm 22.83$ & $88 \pm 21.11$ & 0.823 \\
\hline \multirow[t]{3}{*}{ Type of Surgery (1/2/3/4) } & $12 / 8 / 3 / 2$ & $11 / 6 / 4 / 4$ & 0.828 \\
\hline & ESB Group & Control Group & \\
\hline & $(n=25)$ & $(\mathrm{n}=\mathbf{2 5})$ & $p$ \\
\hline \multicolumn{4}{|l|}{ NRS scores } \\
\hline $1^{\text {st }}$ hour & $2(0.5-3.5)$ & $2(1-4)$ & 0.937 \\
\hline $6^{\text {tn }}$ hour & $2(0-2.5)$ & $2(0-3)$ & 0.386 \\
\hline $12^{\text {th }}$ hour & $0(0-2)$ & $1(0-2)$ & 0.528 \\
\hline $24^{\text {th }}$ hour & $0(0-1)$ & $1(0-1.5)$ & 0.114 \\
\hline \multicolumn{4}{|c|}{ Morphine consumption (mg) } \\
\hline $1^{\text {st }}$ hour & $1(0-1)$ & $1(1-2.5)$ & 0.02 \\
\hline $6^{\text {th }}$ hour & $2(1-4)$ & $5(3.5-7)$ & $<0.001$ \\
\hline $12^{\text {th }}$ hour & $3(2-5)$ & $10(8-12)$ & $<0.001$ \\
\hline $24^{\text {tn }}$ hour & $5(3-8.5)$ & $16(12-19.5)$ & $<0.001$ \\
\hline
\end{tabular}

Conclusion

Our study has shown that US guided ESB has a significant analgesic effect in patients undergoing breast surgery. Further studies, comparing different regional anesthesia techniques are required to search for the optimal analgesia technique for this group of patients.

1. Forero M, Adhikary SD, Lopez Het al. The erector spinae plane block: a novel analgesic technique in thoracic neuropathic pain. Reg Anesth Pain Med 2016;41:621-7

2. Aksu C, Gurkan Y. Ultrasound-guided bilateral erector spinae block could provide effective postoperative analgesia in laparoscopic cholecystectomy in paediatric patients. Anaesth Crit Care Pain Med. E-pub ahead of print.

3. Bonvinci D, Tagliapietra L, Giacomazzi et al. Bilateral ultrasound-guided erector spinae plane blocks in breast cancer and reconstruction surgery. J Clin Anesth. 2017;44:13-4

4. Aksu C, Gürkan Y. Opioid sparing effect of Erector Spinae Plane block for pediatric bilateral inguinal hernia surgeries. J Clin Anesth. 2018 Jul 1;50:62-63 